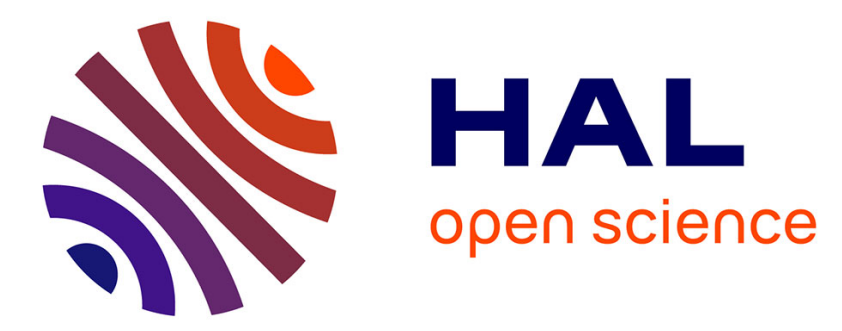

\title{
High-pressure dependent ferroelectric phase transition in lead titanate
}

Nicolas Jaouen, A.C. Dhaussy, Jean Paul Itié, Sylvain Marinel, Yves Joly

\section{To cite this version:}

Nicolas Jaouen, A.C. Dhaussy, Jean Paul Itié, Sylvain Marinel, Yves Joly. High-pressure dependent ferroelectric phase transition in lead titanate. Physical Review B: Condensed Matter and Materials Physics (1998-2015), 2007, 75, pp.224115. 10.1103/PhysRevB.75.224115 . hal-00179001

\section{HAL Id: hal-00179001 https://hal.science/hal-00179001}

Submitted on 15 Nov 2007

HAL is a multi-disciplinary open access archive for the deposit and dissemination of scientific research documents, whether they are published or not. The documents may come from teaching and research institutions in France or abroad, or from public or private research centers.
L'archive ouverte pluridisciplinaire HAL, est destinée au dépôt et à la diffusion de documents scientifiques de niveau recherche, publiés ou non, émanant des établissements d'enseignement et de recherche français ou étrangers, des laboratoires publics ou privés. 


\title{
Nature of the high pressure dependent ferroelectric phase transition in lead titanate
}

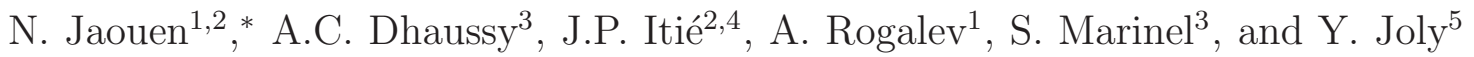 \\ ${ }^{1}$ European Synchrotron Radiation Facility (ESRF), B.P. 220, 38043 Grenoble, France \\ ${ }^{2}$ Synchrotron SOLEIL, L'Orme des Merisiers, 91192 Gif/Yvette, France \\ ${ }^{3}$ CRISMAT, ENSICAEN, Bvd Mal Juin,14050 Caen, France \\ ${ }^{4}$ Physique des Milieux Denses, IMPMC, \\ Univ. P \& M Curie, 75015 Paris, France and \\ ${ }^{5}$ Institut NEEL, CNRS \& Univ. Joseph Fourier, BP 166, 38042 Grenoble, France
}

(Dated: May 29, 2007)

\begin{abstract}
Ti K edge x-ray absorption near-edge structure (XANES) measurements across the pressure induced ferroelectric (FE)-paraeletric ( $\mathrm{PE}$ ) phase transition in $\mathrm{PbTiO}_{3}$ a prototypical ferroelectric perovskite, are reported for the first time. A quantitative analysis of the XANES allows us to obtain the relative local displacement, directly related to the local electric polarization, of Ti and $\mathrm{Pb}$ atoms under pressure. In particular, we found that above the critical pressure, in the so-called cubic-paraelectric phase, the Ti atoms remain locally displaced from their cubic site indicating that this phase transition has in a general way an essential order-disorder component. However the magnitude of these local displacements is strongly affected by the applied pressure as opposed to what happens as a function of temperature. These conclusions have important consequences in the long standing controversial debate on the interpretation of the ferroelectric phase transition of $\mathrm{PbTiO}_{3}$, and related family compounds.
\end{abstract}

PACS numbers: 78.70.Dm, 81.40.Vw, 77.84.-s

Keywords: ferroelectricity, phase transition, high pressure, X-ray Absorption Spectroscopy 


\section{INTRODUCTION}

Perovskites are an extremely important class of ferroelectric materials. Their importance is not restricted to their technological interest, but also more fundamentally to the understanding of structural phase transitions in solids and related symmetry breaking. The perfect $\mathrm{ABO}_{3}$ perovskite structure has a fully cubic symmetry. Thanks to this simple structure, the nature of ferroelectric phase transitions in perovskites has been the subject of intense investigations since the discovery of ferroelectricity in barium titanate. Among these materials, $\mathrm{PbTiO}_{3}$ is a classical example of ferroelectric perovskites. Its temperature-driven cubic to tetragonal phase transition at $\mathrm{T}_{c}=763 \mathrm{~K}$ is taken as a textbook example of a purely displacive transition ${ }^{1-3}$. The origin of this temperature-induced phase transition has been re-opened to debate due to new experimental evidence of the existence of a significant orderdisorder component by using EXAFS $^{4}$, Raman spectroscopy ${ }^{5}$ and x-ray diffraction ${ }^{6}$. This revival and apparent discrepancy between experimental results have been discussed recently for the temperature-induced transition in the case of barium titanate ${ }^{7}$ and related systems.

In 1975, Samara et $a l^{11}$ demonstrated that, unlike temperature, electric field or atomic substitution, pressure is one of the parameters able to control ferroeletricity in perovskites ${ }^{12}$. This reduction can be understood by recalling that external pressure modifies the delicate balance between long-range Coulomb interactions and short range electronic forces, favouring respectively, ferroelectric distortions and a paraelectric cubic structure ${ }^{13}$. Although recent theoretical work predicted either enhancement of the piezoelectricity ${ }^{14}$ or even of ferroelecricity ${ }^{15}$, only a few experimental high pressure x-ray studies of $\mathrm{PbTiO}_{3}$ have been reported with a maximum pressure limited at $8 \mathrm{GPa}^{16,17}$. It is only recently that $\mathrm{x}$-ray diffraction experiments have been successfully carried out under pressure up to $37 \mathrm{GPa}^{18,19}$ in $\mathrm{PbTiO}_{3}$ or related materials ${ }^{8-10}$. The authors ${ }^{18,19}$ report that at room temperature, $\mathrm{PbTiO}_{3}$ has a tetragonal structure $(P 4 m m$ space group a $=3.9036(1) \AA$ and $\mathrm{c}=4.1440(2) \AA)$,

with a decrease of the $\mathrm{Pb}-\mathrm{O}$ and $\mathrm{Ti}-\mathrm{O}$ bond lengths under pressure. At room temperature they show that the tetragonal to cubic phase transition takes place at $11.2 \mathrm{GPa}$ and is of second-order. More interestingly, they conclude that when applying pressure at room temperature the order-disorder contribution is absent or at least strongly reduced. However, the order-disorder component in the phase transition consists of a local displacement from cubic symmetry that partially loses long-range order while the displacive component consists of 
a displacement going to zero at critical pressure. Unless one performs careful analysis of diffuse x-ray scattering, made difficult by the high pressure environment, x-ray diffraction is not the best technique to detect the existence of an order-disorder component. X-ray absorption spectroscopy is a powerful probe of local structure in $\mathrm{ABO}_{3}$ perovskite ${ }^{4,20-23}$ but the extremely severe experimental conditions have prevented studying the pressure induced phase transition at the local scale in $\mathrm{PbTiO}_{3}$ up to now. In this paper, we report on the local structure in the pressure induced decrease of the ferroelectric distortion. Our aim is twofold. First we want to test the general character of the order-disorder component reported in temperature-driven transitions in $\mathrm{BaTiO}_{3}{ }^{4}$ and $\mathrm{PbTiO}_{3}{ }^{22}$. And address the limitations of diffraction using x-ray absorption spectroscopy especially well suited to probe the local displacement of the Ti atoms from their cubic position ${ }^{7}$. Second, it has recently become possible to perform XANES full-potential cluster calculations ${ }^{23}$. So our objective is also to clarify the scenario of this famous phase transition by providing a quantitative estimate of the local $\mathrm{Ti}$ and $\mathrm{Pb}$ displacements relative to their surrounding oxygen polyhedron. According to ${ }^{15}$, antiferrodistortive instabilities, i.e. tilt of the octahedra, are expected to appear for pressure above 20GPa. Therefore in the pressure range reported in our work, we can safely consider only the $\mathrm{Ti}$ and $\mathrm{Pb}$ displacements along the (001) in $\mathrm{PbTiO}_{3}$.

\section{EXPERIMENTAL DETAILS}

Ti K edge measurements were performed in the transmission geometry at beamline ID12 ${ }^{24}$ of the European Synchrotron Radiation Facility. The experimental conditions and the sensitivity of the measurement have already been reported elsewhere ${ }^{25}$ for a reference sample, i.e. $\mathrm{CaTiO}_{3}$, showing no phase transition. Usually the main difficulty in such experiments is that the absorption of the x-rays by the diamond anvils make it impossible to measure the transmitted beam. In our experiment this problem was much more dramatic due to the presence of lead atoms in the sample. In order to reduce this effect we have used thin

diamonds mounted on fully perforated diamond anvils ${ }^{26,27}$. Despite a 1:4 dilution of the sample in boron nitride powder leading to an estimated final transmission of $10^{-6}$ at $5 \mathrm{keV}$, high quality XANES spectra (see Fig. 1.) were successfully recorded for several pressures across the phase transition.

The pre-edge features observed at the $\mathrm{K}$ edge of the $\mathrm{B}$ atom have been shown to be very 
sensitive to the distortion of the local environment around the B atom ${ }^{20,21,28}$. In particular, when the B atoms are displaced from their centrosymmetric positions, an additional peak appears due to transitions into unfilled atomic $d$ level. This feature is far weaker otherwise. This transition from the $s$ to $d$ states is forbidden in the electric dipole approximation and is usually very weak because its existence is only due to a small electric quadrupole matrix element in the absorption cross section. However, the B atom displacement from the cubic site breaking inversion symmetry induces a mixing of the $p$ and $d$ states and thus introduce a large dipole transition. The intensity of this peak is therefore a direct probe of the local displacement of this atom ${ }^{20,28}$.

\section{RESULTS AND DISCUSSION}

Left part of figure 1 shows the XANES spectra of powdered $\mathrm{PbTiO}_{3}$ for several pressures from ambient to $13 \mathrm{GPa}$. If one focuses on the low energy part, the peak already mentioned above, labelled $\beta$, can be observed. But one can also observes several other features, labelled $\alpha, \delta$ and $\delta$ '. The interpretation of these peaks has been widely discussed ${ }^{28-30}$, but a general assessment $^{28,29,31}$ can be given. The $\alpha$ peak is of quadrupolar origin $\left(t_{2 g}\right)$; the $\beta$ peak is dipolar in nature but includes also a small $\left(e_{g}\right)$ quadrupolar contribution; and the peaks $\delta$ and $\delta^{\prime}$ are pure dipolar features. Let us now turn to check how their respective intensities behave under pressure. Starting from ambient pressure, the $\beta$ peak is strongly decreasing up to $5 \mathrm{GPa}$. Finally, above $8 \mathrm{GPa}$, the $\beta$ peak is still slowly decreasing but remains intense up to $13 \mathrm{GPa}$ and a new feature, labelled $\delta$ ', starts to appear and even finishes by dominating the $\delta$ one. Assuming negligible anharmonic vibrations ${ }^{20}$, the strong intensity of peak $\beta$ shows that the Ti atom is still out of the center of the oxygen octahedron in the high pressure phase. This qualitative analysis allows us to dissociate two main regions of interest, the low pressure one (from 0 to $\sim 5 \mathrm{GPa}$ ) and one located close to the critical pressure (11.2 GPa) where the $\delta$ ' peak appears.

In order to quantify the respective $\mathrm{Ti}$ and $\mathrm{Pb}$ local displacement as a function of the applied pressure we performed full potential cluster calculations of XANES spectra. In order to avoid any approximation on the potential shape, we use the finite difference method (FDM) implemented in the FDMNES code ${ }^{32}$. Before going further into the results, let us describe the parameters and the procedure we used for the simulations. The core-hole effect 
on the XANES spectra were taken into account using an excited atom embedded in a cluster containing 146 atoms. In agreement with $\operatorname{Refs}^{23,28}$, the assumption of a completely screened core-hole charge, according to which the $3 d$-shell occupation is increased by one electron, led to a too small separation between peaks $\beta$ and $\delta$. In our simulations we employed a model of incomplete extra atomic screening and we found best agreement when the $3 d$-shell occupation is increased by 0.8 electrons. The calculated spectra were broadened taking into account of the Ti K hole width, photoelectron width and the experimental resolution. For the ambient pressure, the calculations have been performed using the $P 4 \mathrm{~mm}$ space group and the atomic positions reported in Ref ${ }^{16}$. Concerning those for the high pressure, we use as fixed parameters the lattice parameters from $\mathrm{XRD}^{18,19}$ and as starting point the relative $\mathrm{Ti}$ and $\mathrm{Pb}$ local displacement along (001) proportional to the lattice parameters reduction ${ }^{28}$. Then several models for the respective displacement of the $\mathrm{Ti}$ and $\mathrm{Pb}$ atoms were tried.

The left panel of figure 2 shows simulations corresponding to the 2 GPa lattice parameters. We represent the calculated Ti K edge spectra for several models accounting for relative local displacements of the $\mathrm{Ti}$ and $\mathrm{Pb}$ atoms (table 1 ) with respect to their surrounding oxygen polyhedron. It should be noted that we choose to refer to this local displacement as depicted in right part of Fig. 4 rather than the distortion of the polyhedron. We prefer to consider such displacements because they can be directly related to the spontaneous polarization existing locally in the crystal i.e. one of the components of the electric polarization vector. However, this choice results in the same pressure dependence and does not affect the discussion that follows. From this panel, one can clearly see that the $\beta$ peak varies drastically depending on the respective $\mathrm{Ti}$ and $\mathrm{Pb}$ displacements. Even more reliable is the intensity ratio between peak $\beta$ and $\delta$. For example, if one looks at the difference between model 3 and 6 , only a small displacement of $0.03 \AA$ of the $\mathrm{Pb}$ atom towards their symmetric position is sufficient to reduce the $\beta$ peak intensity which becomes equivalent to the $\delta$ one. In addition to illustrate the extreme sensitivity of XANES to a local displacement it also suggest that the error made in our analysis is smaller than $0.03 \AA$. The $\beta$ peak is found to be sufficiently higher than the $\delta$ one only for model 2 in agreement with the experimental data. From this result we conclude that, at $2 \mathrm{GPa}$, model 2 is correct and therefore that the $\mathrm{Pb}$ displacement (15\%) is bigger than the $\mathrm{Ti}$ one (12\%). In that sense, these results reveal that in the low pressure range, the $\mathrm{Pb}$ atoms move faster towards the symmetric position than the Ti ones. It means that for $\mathrm{PbTiO}_{3}$ the contribution of lead atoms is not negli- 
gible in the reduction of ferroelectricity ${ }^{11,12,18,19}$ at low pressure and this has to be related to the importance of lead atoms to stabilize the exceptionally strong ferroelectricity in this compound.

We consider now the pressure range close to the critical pressure. We have already noticed the appearance of an additional feature when going to the high pressure range and the persistence of the $\beta$ one. The right panel of figure 2 exhibits simulated spectra at 13GPa considering various off-center Ti positions along the (001) direction and by keeping the oxygen atoms in cubic positions according to diffraction results ${ }^{18,19}$. For comparison, we show also the theoretical spectra calculated at atmospheric pressure. All the features are correctly reproduced as illustrated by the appearance of the $\delta$ ' peak which corresponds to the hybridization of the empty $p$ states of the absorbing atoms with the unoccupied orbital of the neighbouring atoms. Moreover, according to this figure, the Ti displacement from the centrosymmetric site at $13 \mathrm{GPa}$ can be precisely quantified. Thanks to the high quality of the data and to our simulations, we can estimate a value of $0.09 \pm 0.015 \AA$ for this displacement at a pressure well above the critical pressure. We would like to point out that this remaining displacement is far from being negligible and is close to the one reported at room temperature for the parent compound $\mathrm{SrTiO}_{3}{ }^{33}$. It also supports the view that thermal vibrations alone cannot explain this remaining displacement ${ }^{20}$.

\section{CONCLUSIONS}

In summary, we display in Fig. 3. the XANES simulations made with the same values of pressure that the experimental data of Fig. 1. It allows us to describe and quantify the whole scenario, illustrated in fig. 4, which occurs at the local scale when applying hydrostatic pressure on the ferroelectric prototype compound $\mathrm{PbTiO}_{3}$. From Fig 4. it is clear from the local perspective that the transformation is countinuus. Therefore the dashed line could be used as a guide for eyes for the whole pressure range reported in Fig 4. Thanks to full potential calculations, we are able to extract detailed information on the relative local displacement of $\mathrm{Pb}$ and $\mathrm{Ti}$ under pressure directly related to the local electric polarization. We show that the local displacement of the $\mathrm{Ti}$ atom along (001) in $\mathrm{PbTiO}_{3}$ is still persistent after the pressure phase transition but partially loses this long-range order. Moreover, a precise estimate of this remaining Ti displacement after the critical pressure is given, clearly 
indicating that the existence of an order-disorder component is a general feature in the ferroelectric to paraelectric phase transition in $\mathrm{PbTiO}_{3}$ and related compounds. Interestingly, the reported change in magnitude of local $\mathrm{Pb}$ and $\mathrm{Ti}$ displacements under pressure is clearly bigger than those reported for the temperature-induced phase transition ${ }^{22}$. In other words, this classical phase transition is dominantly an order-disorder one with a dramatic increase of the atomic displacements from the high pressure to the high temperature transition due to different thermodynamic conditions. Let us emphasize that these conclusions have important consequences in the long standing debate on the interpretation of the ferroelectric phase transition for $\mathrm{PbTiO}_{3}$. Moreover, we would like to point out that the recent report of a re-enhanced of the ferroelectricity in $\mathrm{PbTiO}_{3}$ at very high pressure ${ }^{15}$ above the phase transition calls for more experimental work under much higher pressure.

* nicolas.jaouen@synchrotron-soleil.fr

1 G. Shirane and S. Hoshino, J. Phys. Soc. Japan, 6, 256-70 (1951).

2 W. Cochran, Adv. Phys., 9, 387 (1960).

3 B.A. Strukov and A.P. Levanyuk, Ferroelectric Phenomena in Crystals, Springer-Verlag (1998).

4 B. Ravel, N. Sicron, Y. Jacoby, E.A. Stern, F. Dogan and J.J. Rehr, Ferroelectrics 164, 265 (1995).

5 M.D. Fontana, EuroPhys. Lett. 11, 419 (1990).

6 B.D. Chapman, E.A. Stern, S.-W. Han, J.O. Cross, G.T. Seidler, V. Gavrilyatchenko, R.V. Vedrinskii, and V.L. Kraizman, Phys. Rev. B 71, 020102(R) (2005).

7 E.A. Stern, Phys. Rev. Lett. 93, 037601 (2004).

8 J. Rouquette, J. Haines, V. Bornand, M. Pintard, Ph. Papet, W.G. Marshall, and S. Hull, Phys. Rev. B. 71, 024112 (2006).

9 P.-E. Janolin, B. Dkhil, P. Bouvier, J. Kreisel, and P.A. Thomas, Phys. Rev. B. 73, 094128 (2006).

10 M. Ahart, R.E. Cohen, V. Struzhkin, E. Gregoryanz, D. Rytz, S.A. Prosandeev, H.K. Mao, R.J. Hemley, Phys. Rev. B. 71, 144102 (2005).

11 G.A. Samara, T. Sakudo, and K. Yoshimitsu, Phys. Rev. Lett. 35, 1767 (1975).

12 J.A. Sanjurjo, E. Lopez-Crúz, and G. Burns, Phys. Rev. B 28, 7260 (1983). 
13 R. Cohen, Nature (London) 358, 136 (1992).

14 Z. Wu and R.E. Cohen, Phys. Rev. Lett. 95, 037601 (2005).

15 I.A. Kornev, L. Bellaiche, P. Bouvier, P.E. Janolin, B. Dkhil, and J. Kreisel, Phys. Rev. Lett. 95, 196804 (2006).

16 R. Ramirez, H. Vincent, R.J. Nelmes, and A. Katrusiak, Solid State Communication 77, 927 (1991).

17 R.J. Nelmes and A. Katrusiak, J. Phys. C: Solid State Phys. 19, L725 (1986).

18 A. Sani, M. Hanfland, and D. Levy, J. Phys: Condens. Matter 14, 10601 (2002);

19 A. Sani and M. hanfland, J. Sol. State Chem. 167, 446 (2002).

20 J.P. Itié, B. Couzinet, A. Polian, A.M. Flanck, and P. Lagarde, EuroPhys. Lett. 74 (4), 706 (2006).

21 F. Farges, G.E. Brown Jr., J.J. Rehr, Phys. Rev. B 56, 1809 (1997).

22 N. Sicron, B. Ravel, Y. Yacoby, E.A. Stern, F. Dogan, and J.J. Rehr, Phys. Rev. B 50, 13168 (1994).

23 Y. Joly, D. Cabaret, H. Renevier, and C.R. Natoli, Phys. Rev. Lett. 82, 2398 (1999).

24 A. Rogalev, J. Goulon, C.Goulon-Ginet, and C. Malgrange, in Magnetism and Synchrotron Radiation, eds E. Beaurepaire, F. Scheurer, G. Krill, and J.-P. Kappler, Lectures Notes in Physics, vol 565 (2001), Springer.

25 A.C. Dhaussy, N. Jaouen, J.P. Itié, A. Rogalev, S. Marinel and A. Veres, AIP Conf. Proc. 879, 1825 (2007).

26 A. Dadashev, M.P. Pasternak, G.K. Rozenberg, and R.D. Taylor , Rev. Sci. Instrum. 72, 2633 (2001).

27 J.P. Itié, F. Baudelet, A. Congedutti, B. Couzinet, F. Farges, and A. Polian, J. Phys.: Condens. Matter 17, S883 (2005).

28 R.V. Vedrinskii, V.L. Kraizman, A.A. Novakovich, P.V. Demekhin, and S.V. Urazhdin, J. Phys: Condens. Matter 10, 9561 (1998).

29 B. Ravel, E.A. Stern, R.I. Vedrinskii, and V. Kraizman, Ferroeletrics 206-207, 407 (1998).

30 S. Nozawa, T. Iwazumi, and H. Osawa, Phys. Rev. B 72, 121101(R) (2005).

31 J. Danger, P. Le Fèvre, H. Magnan, D. Chandesris, S. Bourgeois, J. Jupille, T. Eickhoff, and W. Drube, Phys. Rev. Lett. 88, 243001 (2002).

32 Y. Joly, Phys. Rev. B. 63, 125120 (2001). 
33 D. Cabaret, B. Couzinet, A.M. Flanck, J.-P. Itié, XAFS XIII Conf. Proc. In Press.

TABLE I: Ti and Pb displacement from symmetric position inside the coordination polyhedra. See Figure 4 for details.

\begin{tabular}{lcc}
\hline displacement $(\AA)$ & $\mathrm{Ti}$ & $\mathrm{Pb}$ \\
\hline model 1 & 0.3 & 0.39 \\
model 2 & 0.28 & 0.39 \\
model 3 & 0.25 & 0.36 \\
model 4 & 0.22 & 0.34 \\
model 5 & 0.28 & 0.42 \\
model 6 & 0.25 & 0.39 \\
\hline
\end{tabular}

FIG. 1: (Color online) Left: Experimental Ti K XANES for $\mathrm{PbTIO}_{3}$ at various pressures from 0 to $13 \mathrm{GPa}$. Right: pre-edge features in details.

FIG. 2: Left: View of the pre-edge region for calculated Ti K edge at 2 GPa with various local displacement of the $\mathrm{Ti}$ and $\mathrm{Pb}$ inside their coordination polyhedron. See table 1 for details. Right: View of the pre-edge region for $13 \mathrm{GPa}$ with various off-center Ti position. $\Delta \mathrm{R}$ is the shift of $\mathrm{Ti}$ along the $<001>$ direction. 
FIG. 3: (Color online) Left: Calculated Ti K XANES for $\mathrm{PbTiO}_{3}$ at various pressures from 0 to $13 \mathrm{GPa}$. Right: pre-edge features in details.

FIG. 4: (Color online)Left: Local displacement of $\mathrm{Ti}$ and $\mathrm{Pb}$ with respect to their coordination polyhedron under pressure. Right: Picture of these displacements. 


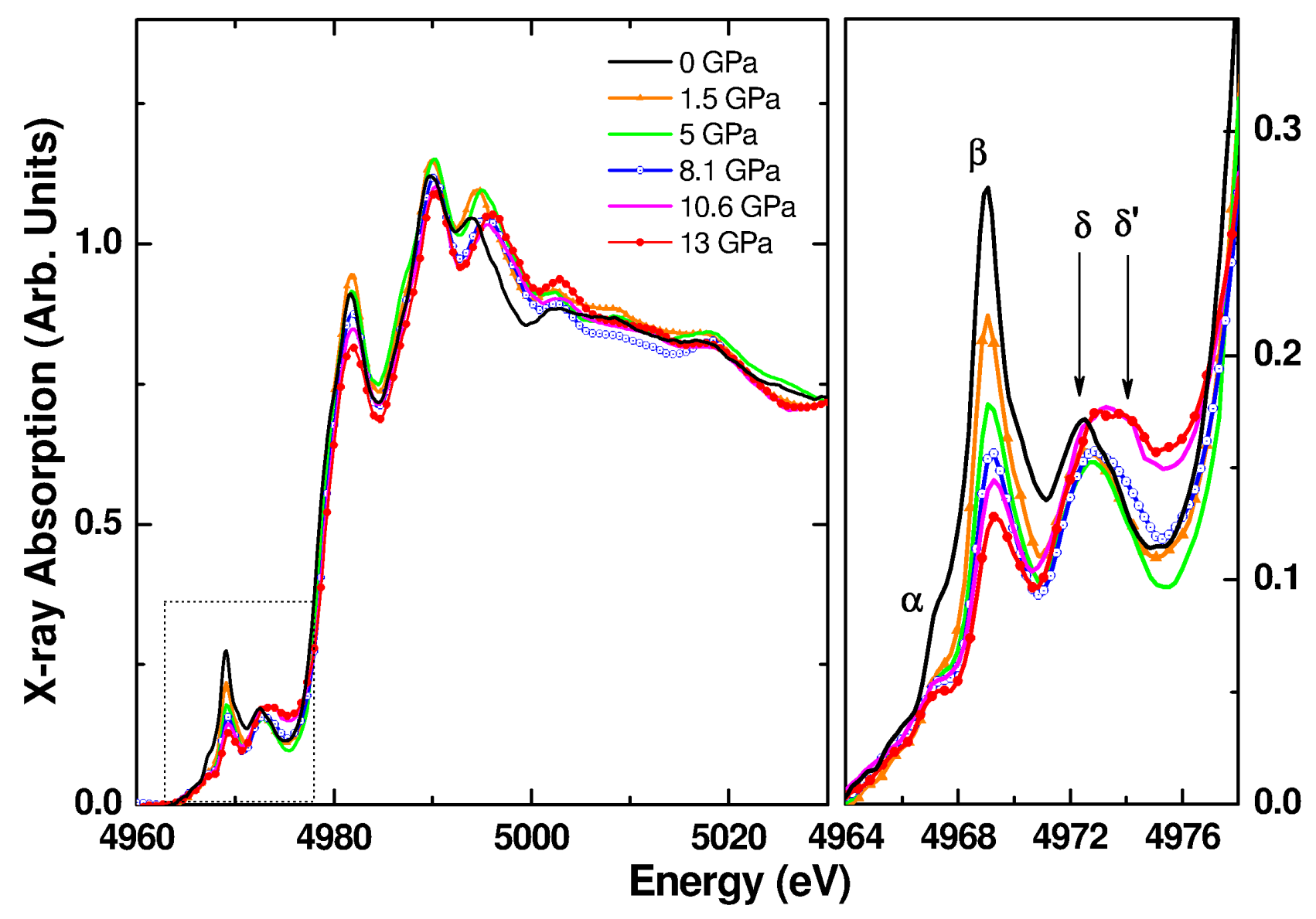

Figure 1 

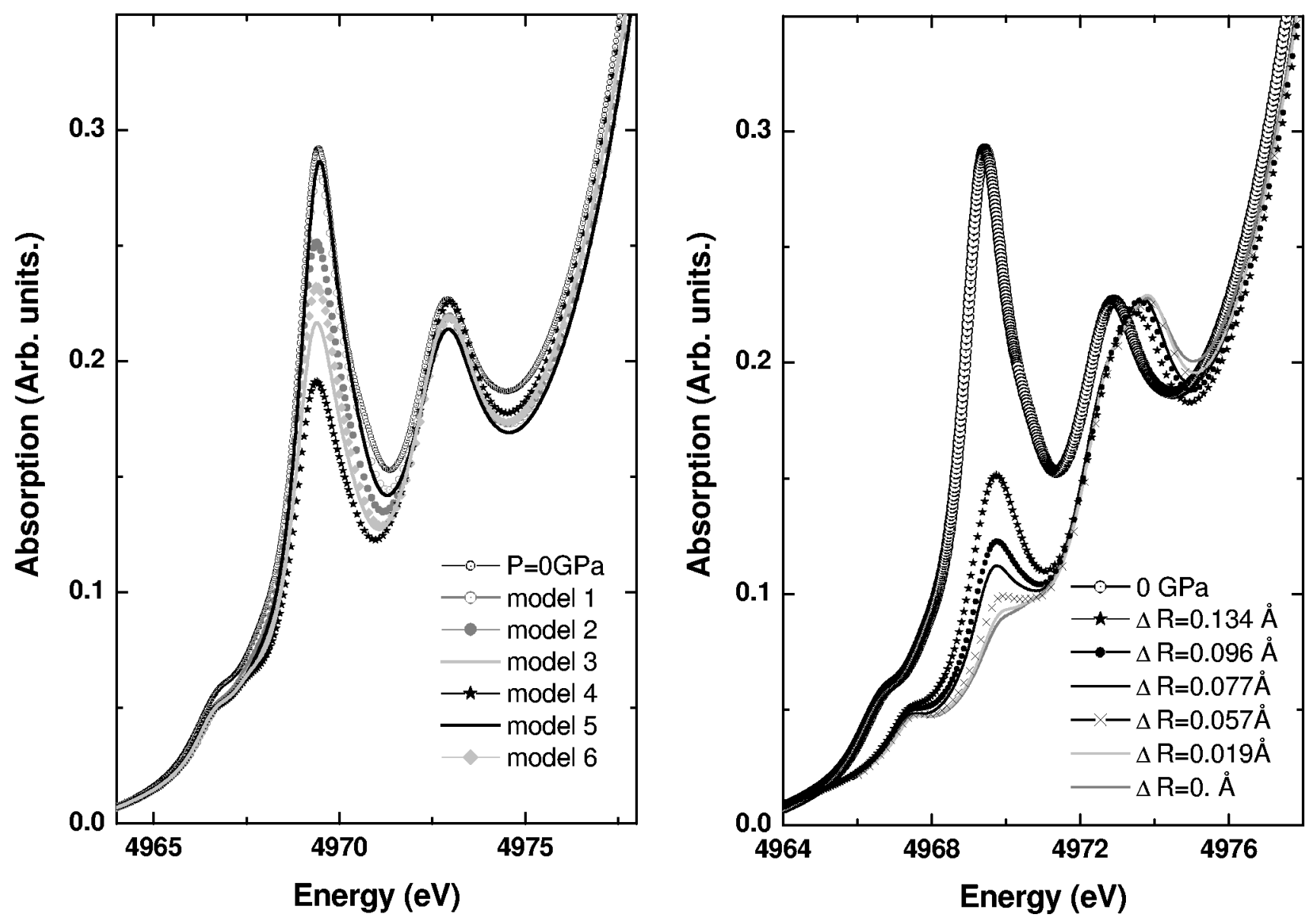

Figure 2 


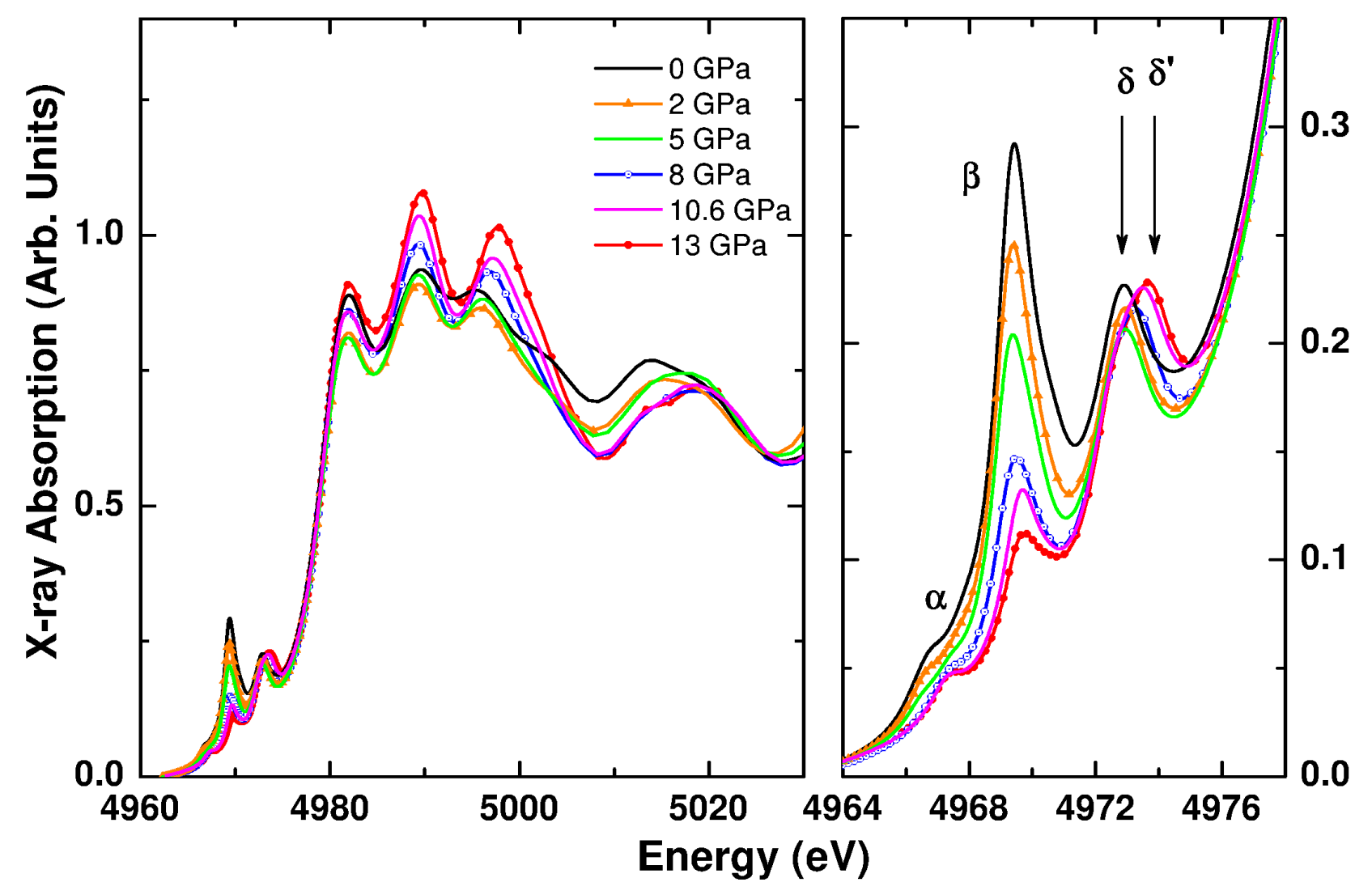

Figure 3 

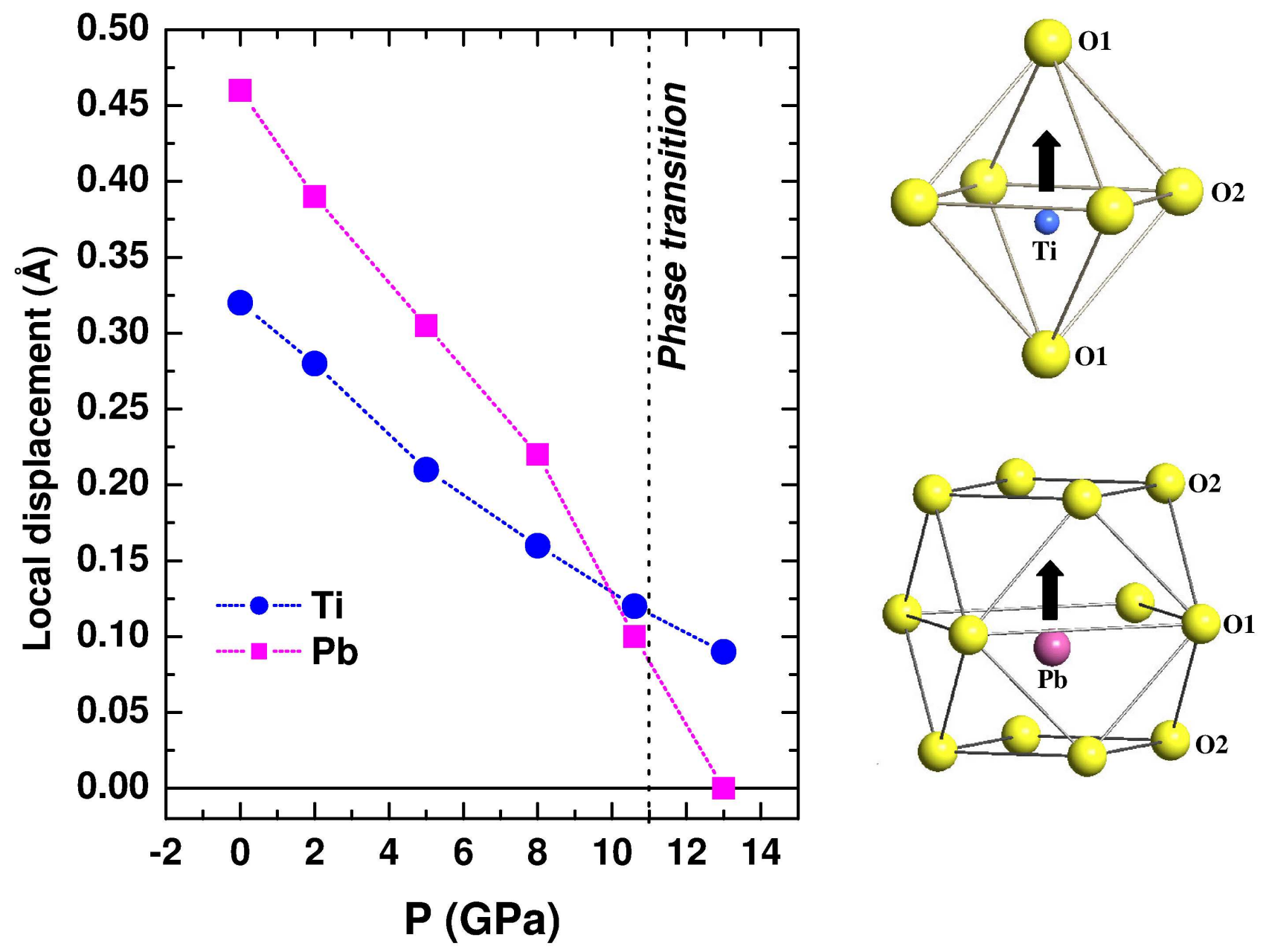

Figure 4 